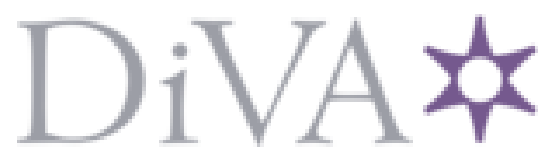

http://www.diva-portal.org

\title{
Postprint
}

This is the accepted version of a paper published in International Journal of Quantum Chemistry. This paper has been peer-reviewed but does not include the final publisher proof-corrections or journal pagination.

Citation for the original published paper (version of record):

Brändas, E. (2014)

The Statement of Goals of the International Society forTheoretical Chemical Physics.

International Journal of Quantum Chemistry, 114: 961-962

http://dx.doi.org/DOI: 10.1002/qua.24712

Access to the published version may require subscription.

N.B. When citing this work, cite the original published paper.

Permanent link to this version:

http://urn.kb.se/resolve?urn=urn:nbn:se:uu:diva-238711 


\section{The Statement of Goals of the International Society for Theoretical Chemical Physics}

In recent years there has been important progress in the theory of chemical phenomena with its well-established roots built on basic physical principles. As a result of this commitment, the domain of theoretical chemical physics has evolved to embrace entrenched fundamental knowledge. In particular the main themes of Theoretical Chemical Physics, have attracted considerable attention owing to the general developments of theoretical methodologies as well as to significant advances in computer technologies. Theoretical Chemical Physics has become an indispensable resource for carrying out fundamental scientific research. Recent work has not only incorporated theoretical chemical physics, with reference to dynamics, and statistical mechanics but also included principal applications from quantum chemistry to material science, biochemistry, biophysics and even medicine, the latter being recently highlighted by the Nature supplement: Physical Scientists Take On Cancer.

The present situation is indeed exceptional, not only since the challenges posed by quantum chemistry have motivated and inspired several generations of scientists, but most importantly have lead to an enormous software development bringing robust, fast and accurate tools to the community of method consumers and developers. The possibility for various groups to selforganise around particular platforms, with existing packages from a playground of testable ideas to black-box chemistry codes of the large-scale, thereby providing indispensable and vital infrastructure for scientific contributors, is most probably unmatched in any other discipline.

More specifically the subjects involve:

1. Concepts and Fundamental Methods in Chemical Physics

2. Frontiers in Quantum Chemistry, Wave Functions and Density Functional Theory

3. Frontiers in Molecular Dynamics and Molecular Simulations

4. Computational Quantum Chemistry and Chemical Physics

5. Applications to Atomic and Molecular Structures, Properties, and Spectroscopies

6. Applications to Chemical Reactions and Dynamics

7. Applications to Structures, Properties, and Functions of Complex Systems

8. Applications to Biochemistry and Biophysics

9. Applications to Meso- and Nano-Science

The present definition of Theoretical Chemical Physics and its domain at large calls attention to fundamental questions and invites general queries. For instance, even if it is, in many cases, possible to compare, with high precision, theory with experiments, the macroscopic description does not always correlate appropriately with the corresponding microscopic representation. For instance, the portrayal of the latter is indeed in obvious contrast with the former, e.g. in connection with questions of unidirectional time and time invariance and with related derivations of irreversible thermodynamics from statistical mechanics. These issues are interconnected in the most complex fashion and for this reason critical with respect to our primary understanding of the world we live in. As an illustration we refer to the fundamental age-old dilemma, whether the second law of thermodynamics is an absolute constitution of nature or not. 
There is moreover an accumulating amount of modern chemical phenomena, where the detailed dynamics is in demand of far-reaching theoretical models and conceptions, i.e. the significance and construal of the presence of the (so-called) unstable states in the continuous spectra. In this domain one finds e. g. primary investigations regarding time-resolved effects of both nuclear and electronic motions motivating the notion of a new age of quantum chemistry. The maturity of the field has already led to the coinage of Non-Hermitian Quantum Mechanics as an alternative to the standard formalism of Quantum Mechanics.

Since, as portrayed, (the theory of chemistry) Theoretical Chemical Physics comprise many different branches, it is understandable that the communication between scientists working in the various subfields needs to be constantly updated and perfected. From the beginning the main goal of the proposed Society has been to establish a forum for researchers working in these different disciplines to meet at international-, regional- and national meetings (congresses, conferences, symposia, workshops, etc.), which the Society is going to organize and support through its interactions and representations in the scientific community. The interdisciplinary, yet fundamental, nature of chemical physics, that lies behind the objectives of the Society should lead by crossfertilization to original results and innovations thereby strongly promoting the growth and progress of the whole scientific field.

In this day and age, when information and communication are overflowing particularly due to the rapid development of internet-supported services creating highly interacting platforms one may reflect on whether one needs a traditional society, like ISTCP, for promoting Theoretical Chemical Physics. With facebook, twitter and various types of blogs, a social networking site should perhaps be better and with higher intensity more profitable. ISTCP, however, has existed for nearly 25 years, which is a time scale that far exceeds those of recent web-based technologies. It is hence more important to build up and fuse together the themes of Theoretical Chemical Physics at a less intensive rate with goals and ambitions that aim at solving unforseen problems and confronting the demands of the future. It is in this mould that ISTCP finds its most profound challenges.

Erkki Brändas

President ISTCP 